\title{
Conservación de medallas en las bibliotecas
}

\author{
Ana Luisa Figueredo Figueredo \\ Universidad de Granma - UDG, Cuba \\ Dianelis Lázara Borrego Zaldívar \\ Unidad Empresarial de Base de Información Científico Técnica AICROS - UEB - ICT aicros, Cuba
}

\section{REVIEW}

\section{Resumen}

La conservación de medallas tanto en museos como en bibliotecas nacionales, universitarias y otros sistemas de información que atesoran este tipo de objetos, ha sido ampliamente discutida entre los especialistas y conocedores del tema durante muchos años. En este trabajo se abordan elementos de las medallas que las identifican como documentos y se esbozarán elementos mínimo-indispensables para asegurar la adecuada conservación de este tipo de colección en aquellos lugares donde no es muy frecuente encontrarlas como las bibliotecas.

Palabras-clave

Conservación de medallas ; Bibliotecas ; Colecciones de medallas

\section{Conservation of medals in libraries}

\section{Abstract}

Conservation of medals, either in museums or national and university libraries, as well as in other information systems that store these objects, has been widely debated among scholars and experts on the subject for many years. This paper deals with the elements that identify medals as documents. More over, minimum-essential elements are outlined to ensure an adequate conservation of this type of collection in places where they are rarely found such as libraries.

Keywords

Conservation of medals ; Libraries ; Medals collections

\section{Introducción}

Históricamente las bibliotecas, independientemente de su tipología, han sido consideradas como el depósito para atesorar, conservar y difundir información registrada, principalmente en forma impresa o manuscrita. Sin embargo, su acepción moderna, y desde hace ya algún tiempo, incluye cualquier tipo de información recogida en diferentes formatos: grabaciones, películas, diapositivas, microfilmes, revistas, cintas magnéticas y de vídeo y otros. Incluso, su universo se ha extendido hasta el punto de contener colecciones, que durante mucho tiempo fueron tratadas sólo en museos, fundamentalmente colecciones de objetos creados por el hombre.

Muchas de ellas han pasado a formar parte sustancial de grandes bibliotecas nacionales, públicas y universitarias. Algunas de estas bibliotecas desde su propia creación contaron con colecciones de esta naturaleza y las fueron 
enriqueciendo a través de donaciones y compra de colecciones particulares, el canje con diferentes instituciones culturales, y en muchos casos por lo establecido con el Depósito Legal en cada país.

Dentro de estas colecciones, las numismáticas han despertado el interés de un gran número de bibliotecas en todo el mundo y especialmente las monedas y medallas.

Conservar estos archivos, que no son perecederos como el papel, es deber cultural; generalmente los investigadores vuelcan sus nobles afanes en las letras manuscritas e impresas, olvidando que existen otros materiales de naturaleza intrínseca, bien distintos, que complementan o comprueban aquellos en no pocas ocasiones y que son materia de investigación y estudio, que sujetos al análisis y a la crítica pueden brindar útiles informaciones (Academia Nacional de Medicina de Buenos Aires, p.21)

\section{Antecedentes}

Una medalla es un testimonio que se deja para el futuro, como reconocimiento a un acontecimiento digno de recordarse, el premio a un esfuerzo personal o de alguna institución, por alguna labor realizada o como constancia de su iniciación en alguna actividad, en fin, hay múltiples motivos para los que se acostumbra hacer medallas y las personas e instituciones públicas o privadas, tienen toda la libertad de planearla y hacerlas como quieran, pero siendo parte del patrimonio nacional del futuro.(Federación de Numismáticos Colombianos, p.37)

Muchas de las investigaciones realizadas a lo largo de la historia, se han apoyado para su desarrollo en los documentos impresos y manuscritos; sin embargo existen muchos objetos tanto naturales como creados por el hombre, que constituyen importantes materiales de investigación, convirtiéndose en ocasiones, en los únicos testigos de un acontecimiento (Domínguez, p.116), o completando aquello que aparece recogido en otro tipo de soporte.

En nuestros tiempos ha venido a aumentar el número de fuentes de información que forman parte de los sistemas de información, incluso algunas en las que tiempo atrás no se pensaba; así como la incorporación de tratamientos de procesamiento, almacenamiento, diseminación y uso, correspondiente a cada tipo.

La Documentación fundada formalmente en 1934 contribuyó al enriquecimiento de las consideraciones sobre lo que debía ser visto como documento. Otlet plantéo "Toda fuente de información física que comunicase inscripciones, imágenes, sonido, texto, objetos con indicios de intervención humana, creaciones artísticas e incluso materiales naturales".(Otlet p.16)

A esta definición sucedieron otras que siguieron la idea planteada por Otlet, como la de Mijailov "Cualquier objeto material que registre o fije algún conocimiento y pueda ser incluido en una recopilación" (Mijailov, Chernii y Guiliarovskii, 79 )

No limitaban los autores el documento a las letras impresas o manuscritas, sino que incluían obras que atesoraban los museos, independientemente de su naturaleza.

Según Andrés Cruz Paz "documento puede ser cualquier fuente de información, en forma material, capaz de ser empleada para referencia o estudio o como una autoridad" (Cruz, 268)

Tomando como referencia estas definiciones se puede comprobar que la importancia de un documento radica más en la función que este desempeña, que en su forma física. Con la diversificación de las tipologías de documentos que se seleccionan, organizan, preservan, y diseminan en las bibliotecas: medallas, monedas, pinturas, grabados, tarjetas postales, mapas, grabaciones sonoras y audiovisuales, se incorporan nuevas normas, reglas y técnicas de procesamiento y conservación, para dar un tratamiento adecuado a cada documento.

Equívocamente, y en la mayoría de los casos por desconocimiento, se considera que tanto las medallas como las monedas por estar fabricadas en metal, no requieren determinados cuidados para su conservación a través del tiempo, debido a que este material es menos sensible que otros como el papel, soportes ópticos (videodiscos, CDROM, discos compactos), o magnéticos (cintas magnéticas y de video, disquetes); sin embargo, resulta todo lo contrario. Además de esta consideración errónea en cuanto a la atención que deben recibir estos objetos metálicos, se incorporan como factores que dificultan la conservación: el número de ejemplares, las dimensiones (pequeñas) y la "aparente" facilidad de almacenamiento. 
Esta conservación, en una de sus tantas acepciones se refiere al: "medidas para proteger una información en diferentes soportes, con el objetivo de que perdure durante un período prolongado".(Mirabal)

Las medallas necesitan de condiciones y tratamientos adecuados para su conservación, que al igual que la de otros objetos metálicos resulta particularmente compleja. Hay que tener en cuenta, además, que ellas son dentro de las piezas numismáticas, las más difíciles de conservar, ya que muchas se encuentran acompañadas de otros componentes, como las telas, que forman parte de la pieza y los esmaltes, cuyo deterioro perjudica su conservación. Esto determina que deba prestársele atención a ambos materiales (si es el caso), a la hora de tomar cualquier decisión relacionada al tema.

A continuación se mencionarán algunas bibliotecas que atesoran colecciones de medallas. En algunos casos se darán algunas de sus características fundamentales como parte del fondo documental de estas instituciones, pues no todas facilitan información al respecto.

- Biblioteca Pública Universidad Nacional de la Plata

Esta colección de la sección numismática está integrada por 254 piezas, conservadas en la Biblioteca a partir de 1903, por gestión del entonces Director Sr. Luis Fors. Diversas circunstancias han determinado que durante algún período se depositaran en el Museo de Ciencias Naturales. Desde 1983 permanecen en forma definitiva en esta Biblioteca Pública, donde han sido reacondicionadas y organizada la información acerca de sus características. (Biblioteca Pública Universidad Nacional de la Plata)

- Biblioteca Británica

Tiene entre sus departamentos, uno dedicado a las monedas y medallas, que abarca todas las culturas y períodos de la historia de la humanidad.

Su material numismático incluye piezas griegas, romanas y orientales, así como la Colección Real presentada al Museo por Jorge IV. Reúne una colección de medallas conmemorativas y artísticas, de acontecimientos históricos y escuelas artísticas desde el renacimiento italiano hasta el siglo XXI.

- Biblioteca Apostólica Vaticana

La biblioteca atesora alrededor de 300000 medallas y monedas. La catalogación automatizada de las medallas incluye notas de referencia de todo lo relativo al tema, especialmente información de las que se preservan en la biblioteca.

Con esta informatización se adoptaron las Reglas de Catalogación Angloamericanas, pero conservando las Normas Vaticanas de encabezamientos de materias. El Gabinete Numismático o Medagliere de la Biblioteca Vaticana conserva monedas, medallas, placas, sellos y otros documentos.( Biblioteca Apostólica Vaticana)

- Biblioteca Nacional de Francia

La Biblioteca Nacional de Francia es considerada como una de las mayores y más moderna biblioteca del mundo, albergando la mayor parte del patrimonio de Francia.

Los edificios de la actual biblioteca fueron rehabilitados para albergar parte de las colecciones de la antigua Biblioteca Nacional: manuscritos, láminas, fotografías, mapas y planos, partituras de música, monedas, medallas antiguas y elementos relativos al espectáculo, libros, el material impreso y los documentos audiovisuales.

Dentro de sus departamentos, el destinado a las monedas, medallas y antigüedades, se le conoce por el nombre de "Gabinete de medallas" o "Gabinete de Francia". Este nació de la colección de los reyes de Francia durante la Edad Media. Desde su surgimiento se enriqueció a través de la donación de colecciones particulares de príncipes, bibliófilos y 
coleccionistas numismáticos. (Biblioteca Nacional de Francia).

Además de la donación, la compra y el depósito legal, son otras de las vías de adquisición de las piezas. En esta última, la Casa de la Moneda de París tiene una gran importancia.

- Biblioteca Nacional de Colombia

Reúne libros y folletos ingresados por depósito legal, compra, canje, donación, y publicaciones de organismos internacionales, publicados principalmente desde 1830; así como grabaciones sonoras, video - grabaciones, películas, archivos de computadora, material cartográfico, material gráfico, microformas, artefactos tridimensionales, kits y materiales no estandarizados.

Las medallas que atesora la biblioteca, se encuentran formando parte de su Fondo Especial, y no como una colección independiente. Este, a su vez, esta integrado por colecciones particulares pertenecientes a personalidades colombianas, adquiridas por donación o compra, de las que formaban parte estas piezas.

La organización y clasificación adoptada por la Biblioteca para cada tipo de obra, está en dependencia del tipo de colección definida.

\section{Metales usados en la fabricación de medallas}

Hay características que son comunes para todos los metales y otras que no lo son, y que en conjunto han permitido al hombre investigar e interesarse cada día más por este fascinante mundo, que es tan variado como complejo. Cada uno de ellos tiene, características que los diferencian entre sí, y por consiguiente no todos deben estar sometidos a los mismos métodos de conservación, sino que estos se adecuan a los metales o las aleaciones en cuestión y las condiciones físicas en que se encuentra. Entre los materiales más usados para su fabricación tenemos: plata, bronce, cobre, aluminio, latón, calamina, estaño.

Hay características que aunque tienen sus excepciones, las comparten casi todos los metales. Estas pueden ser ventajosas o no en dependencia de la aplicación que se le quiera dar. Algunas de ellas son: alto punto de fusión, rigidez, resistencia y durabilidad, formabilidad, ductibilidad reacción con los agentes químicos y conductividad térmica y eléctrica elevada.

Uno de los mayores peligros a los que están sometidos estos metales es a la corrosión. La corrosión se desarrolla en detrimento del metal de que está hecho el objeto. Habrá dos tipos de corrosión: la que se produce sobre la superficie original del objeto y la que se produce por debajo de ella. Así pues, independientemente de los accidentes mecánicos que pueda sufrir el objeto, por efecto de la corrosión aumentará el volumen y perderá más o menos su color, su forma, su peso, su materia y su resistencia. En último término, el objeto corroído se torna ilegible si no se somete a tratamientos particulares.( France_Lanord, p.63)

\section{Condiciones de almacenamiento}

Es de extraordinaria importancia para la adecuada conservación de estas piezas, tener en consideración las condiciones del almacenamiento.

Para prevenir el deterioro de las piezas ubicadas tanto en las reservas como en las salas de exposición, internacionalmente se diseñan y construyen estructuras especiales. En cuanto al sistema de almacenamiento a emplear hay que tener en cuenta si los materiales con los cuales está confeccionado poseen sustancias que puedan afectar la pieza contenida en él. Cada metal tiene sus características propias, que los hace reaccionar de forma diferente ante la exposición a determinadas sustancias, en determinados ambientes y bajo ciertas condiciones.

Existen una gran variedad de sistemas en los cuales pueden almacenarse las medallas. Dentro de estos los más utilizados son: álbumes con hojas de plástico, bandejas de plástico, sobres (de papel, cartón, plástico, celofán), monetarios, cápsulas, entre otros, adaptado cada uno a las características y condiciones físicas de las piezas, las necesidades y posibilidades del coleccionista. 
A la hora de elegir el sistema más adecuado a nuestras necesidades y características de la colección o de las instalaciones, hay que tener en cuenta una serie de aspectos que a la hora de trabajar con la pieza nos facilitarán la labor y que son, entre otros: la seguridad, accesibilidad, visibilidad e inalterabilidad.(Feria, p.31)

Cuando son de plástico, generalmente no se corroen como los materiales metálicos sino que se degradan o deterioran a causa del hinchamiento, descascaramiento, decoloración, envejecimiento, ablandamiento y pérdida de sus propiedades. Todas estas alteraciones pueden afectar la conservación de las medallas, al exponerse a estos tipos de reacción.

Para las maderas se aplican revestimientos que protejan las piezas de sustancias presente en algunas de ellas. Además, hay que tener en cuenta que esos recubrimientos no reaccionen con los metales de las piezas.

Al igual que los monetarios, las bandejas pueden estar fabricadas de madera y plástico, lo que conlleva a tomar las precauciones mencionadas para el sistema anterior. Los sobres de papel y cartón deben estar confeccionados con sustancias neutras.

El vidrio es uno de los principales componentes de las vitrinas y no causa afectaciones a las piezas. El resto de los componentes que conforman la estructura de este sistema: madera, plástico, metales y adhesivos sí deben ser objeto de atención.

En las salas de exposiciones lo deseado para la exhibición de las piezas son las vitrinas con las características adecuadas que eviten la acumulación del polvo, variación de las condiciones atmosféricas y que estén hechas con materiales neutros que no afecten las piezas en exposición.

Un almacenamiento inadecuado es la oportunidad ideal para que la corrosión, producto de su interrelación con el medio ambiente pase a desempeñar su papel fundamental: el deterioro de la pieza.

Todos los sistemas tienen sus ventajas, y ya sea con el empleo de uno o de otro, lo que se persigue es lograr las condiciones adecuadas de almacenamiento y a su vez garantizar que no aparezcan procesos de degradación de las piezas.

\subsection{Manipulación de las medallas}

Es otro de los factores que puede actuar de forma negativa sobre las piezas si no se cuenta con la destreza necesaria, por lo que se recomienda que deben ser manipuladas lo menos posible. Con el objetivo de contribuir a mejorar la conservación de las medallas, esta pasa a desempeñar una función muy importante. Es necesario tomar un conjunto de medidas en las que se tenga como punto de partida para su aplicación, el material del cual esta hecho el objeto, en este caso el o los metales que componen la medalla, así como su forma y hasta que punto sus características permiten su manipulación. Dentro de estas medidas, que hay que poner también en conocimiento de los usuarios, se encuentran: tener destreza para manipular las piezas, evitar colocar algún tipo de numeración, codificación o cualquier aditivo que deje sobre ellas marcas que ocasione la pérdida del valor numismático de la pieza y la información que posee, no poner las medallas en contacto con objetos que las puedan rayar o marcar, como por ejemplo cuchillos, agujas, no se deben agrupar las medallas una encima de la otra, así como dejarlas bajo una luz directa (natural o artificial) durante un largo período, entre otras.

\section{Limpieza}

La limpieza es una operación que no puede ser tomada a la ligera, sino que por el contrario debe ser realizada con extremas precauciones. Una limpieza mal hecha puede ocasionar alteraciones serias a las medallas y tornarlas ilegibles, perdiendo, de esta forma, el mensaje del cual son portadoras y en el peor de los casos la destrucción de la pieza.

Es importante saber diferenciar entre la suciedad y las características que van tomando las piezas con el tiempo y que se convierten, en detalles exclusivos de cada una, dándole belleza a las medallas. 
Muchos de los daños ocasionados no son visibles en el momento de la limpieza, ni a corto plazo, sin embargo esto no quiere decir que en el futuro la pieza no sufra las consecuencias de una decisión incorrecta.

La limpieza, cuyo fin es hacer legible el objeto para restituirle su significación, abarca la primera serie de operaciones. Consiste en limpiar la corrosión que se formó en la superficie primitiva del objeto y en conservar y estabilizar la que se formó por debajo de ella. Es siempre una operación delicada porque es irreversible; muchos objetos han sido más o menos alterados o destruidos por una limpieza mal hecha.( France_Lanord, p.67-68)

La limpieza repetida de la pieza, aumenta el efecto de abrasión, es decir, desgaste en la superficie, pérdida de color, y en general se modifican sus características originales. Se recomienda que ésta operación se realice una sola vez.

De la misma forma que puede contribuir a estabilizar sus condiciones físicas y evitar grandes consecuencias, al mismo tiempo puede ir eliminando en cada ocasión que se realice, detalles que para alguien que no sea conocedor de la materia pasen inadvertidos o simplemente no le den la debida importancia.

Por estas razones, se recomienda que los procedimientos de limpieza sean decididos y realizados por especialistas suficientemente capacitados y no llevarlos a cabo de manera improvisada para evitar daños de carácter irreversible.

\subsection{Factor humano}

El personal responsable de la conservación es otro de los factores importantes de este proceso, que debe mantener en todo momento una posición activa con todo lo relacionado a la colección y su entorno. Debe estar en constante superación profesional, interesarse por los cambios que ocurran en su medio y qué le aportan a su trabajo en dependencia de la institución donde se desempeña y las características de las colecciones que atiende. Debe ser celoso con el cuidado de ésta e incansable en la búsqueda de soluciones y alternativas ante las dificultades que puedan surgir.

\section{Conservación preventiva - restauración}

A pesar de que la conservación es un proceso independiente al de la restauración, ambos guardan estrecha relación y son determinantes en cuanto a lograr la perdurabilidad de las piezas a través del tiempo.

Antes de aplicar cualquier técnica de conservación, no se debe pasar por alto las recomendaciones del Instituto Internacional de Conservación, donde se señalan elementos como: el respeto a la integridad del objeto, competencia del conservador y facilidades para el trabajo, consideración del objeto como modelo único, adecuación del tratamiento, reversibilidad del tratamiento, limitaciones sobre la integridad estética, autoeducación continúa, disponer del personal auxiliar.

Con relación al proceso de restauración se quiere destacar que los daños ocasionados a una medalla o moneda es en extremo una tarea difícil, delicada y costosa. Por esto se insiste tanto en la necesidad de tratar de evitar posibles daños antes de que estos ocurran, es decir, llevar a cabo una conservación preventiva.

El término conservación preventiva, como el mismo indica, se refiere a evitar el daño antes de que ocurra, lo que debe ser objetivo fundamental de cualquier institución o grupo de individuos en el deber de preservar una colección. El daño siempre es irreversible por exquisita que llegue a ser una restauración_a la que generalmente se llega cuando la conservación preventiva no ha sido adecuada.(Morales, p.15)

Según lo planteado por Guichen, "Ia conservación preventiva implica cambiar la antigua mentalidad para que el objeto de ayer se convierta en la colección de hoy, la sala en el edificio, el individuo en el equipo, el presente en futuro, los profesionales en el público en el más alto sentido de la palabra, el secreto en comunicación y el cómo en el por qué". (Guichen, p.15)

Uno de los primeros elementos a tener en cuenta para esta conservación preventiva es determinar en que condiciones físicas se encuentra la colección.

No se limita esta conservación preventiva sólo a lo concerniente a las colecciones en particular. Incluye, además, todo lo referente al edificio y salas (de reserva o exposición): su ubicación topográfica, iluminación, temperatura, humedad y todo aquello que pueda influir de forma negativa sobre la conservación de las medallas. Implica, también, 
todo lo que debe realizar el personal responsabilizado en el tratamiento de las colecciones en función de éstas y de la instalación.

Llegar a determinar el grado de conservación de una medalla siempre está sujeto a la subjetividad del hombre. Pero lo que si es un hecho es que para alcanzar las habilidades necesarias, se requiere de tiempo con experiencia acumulada. Esta se alcanza a través de la observación del mayor número de piezas posibles, informándose sobre el tema mediante consultas a especialistas u otros coleccionistas, así como la lectura. Todo esto con el objetivo de llegar a valoraciones propias con relación a la colección.

Recordemos, para finalizar, que muchas medallas datan de siglos de existencias. Si a esto se le añade que muchas son testimonios únicos sobre acontecimientos extraordinariamente importantes, hará tomar conciencia que si sólo pensamos en una conservación para el presente, en el futuro no se tendrá memoria alguna de aquello que ocurrió en el pasado y que las convierten en fieles portadoras de ese mensaje.

Philip R. Ward asegura que "todo lo que realmente conocemos sobre nosotros mismos y sobre nuestro mundo proviene del pasado. $Y$ todo lo que conocemos verdaderamente del pasado es aquella parte que ha sobrevivido bajo la forma de objetos materiales. Solamente una pequeña fracción de nuestra historia está consignada en la literatura y la literatura está sujeta a los errores de la interpretación humana. Sólo los especimenes materiales de la historia natural y humana son indiscutibles, ya que son la materia prima de la historia, los hechos innegables, la verdad sobre el pasado. La conservación es el medio a través del cual lo preservamos. Es un acto de fe el futuro" (Ward, p.7)

\section{Conclusiones}

Este es un tipo de colección especial que conservan un número reducido de bibliotecas en todo el mundo.

Independientemente del soporte, deben ser tratadas como un documento más, formando parte del acervo cultural de cada país y de estos sistemas.

Aunque cada día aparecen nuevos métodos y procedimientos con importantes aportes en la materia de conservación de estos documentos, es sin lugar a dudas, la conservación preventiva la mejor opción para su preservación.

\section{Bibliografía}

ACADEMIA NACIONAL DE MEDICINA DE BUENOS AIRES (1972). "Exposición de Medalla de Medicina". Buenos Aires: Instituto Bonaerense de Numismática y Antigüedades. p.21.

BIBLIOTECA APOSTÓLICA VATICANA (2012). Disponible en: http//www.vaticanlibrary.va. Consultado 21 nov. 2012.

BIBLIOTECA NACIONAL DE FRANCIA (2012). Disponible en:

http//www.bnf.fr/fr/fr/collections et services/collections departements. Consultado 21 nov. 2012.

BIBLIOTECA PÚBLICA UNIVERSIDAD NACIONAL DE LA PLATA (2012) Disponible en:http://biblio.unlp.edu.ar/new/col esp.html. Consultado 23 nov. 2012.

CRUZ PAZ, A (1992). "En torno a los conceptos de documento, fuente y recurso en la Ciencia de la Información". Ciencia de la Información 23(4) diciembre: 268.

DOMÍNGUEZ, A. "El papel de la Numismática en el conocimiento histórico. Distintas concepciones de la moneda". En: Asociación Numismática Española(1989). Gaceta Numismática (Barcelona)94-95 III/V. sept_dic. p.116.

FEDERACIÓN NUMISMÁTICOS COLOMBIANOS (1997). Boletín Numismático. Año 34 (enero_junio). p.37 
ए10 0 Conservación de medallas en las bibliotecas

FERIA, R. ARCHIVO Y EXPOSICIÓN. En: VIII Congreso Nacional de Numismática: Memorias. 1_4 abril; 1992 p. 31.

FRANCE_LANORD, A (1982). "Metales y tratamientos peligrosos". Museum. Vol 34 (1). p.63_64.

MORALES, I (2002). "Conservar: un compromiso con el futuro". Boletín Numismático No 1 enero_marzo. p. 15

Metales y tratamientos peligrosos. Museum. Vol 34 (1); 1982. p.63

GUICHEN, G(1999). “La Conservación preventiva: ¿simple moda pasajera o cambio trascendental?” Museum Internacional (Paris) 51(1): 15.

MIJAILOV, A. I, CHERNII Y R. S. GUILIAROVSKII (1973). Fundamentos de la Informática. La Habana: Academia de Ciencias de Cuba : Instituto de Documentación e Información Científica y Técnica. t. 1, p. 79.

MIRABAL, M. En: Mesa, D (2011). “Memoria histórica: Disco duro de la nación”. Alma Mater. 49 (9) Mayo.

OTLETT, P. "Traité de Documentación". En: Moreiro González, J A(2001). Introducción al estudio de la información y la documentación. La Habana: [s.n.].p. 16.

WARD, P R(1982). “La Conservación: el porvenir del pasado”. Museum (París) 34(1 abril): 7.

\section{Datos de las autoras}

Ana Luisa Figueredo Figueredo

Licenciada en Bibliotecología y Ciencias de la Información. Máster en Ciencias de la Educación Superior. Profesora instructora de la Universidad de Granma.

afigueredof@udg.co.cu

Dianelis Lázara Borrego Zaldívar

Directora de la Unidad Empresarial de Base de Información Científico Técnica AICROS.

dianelis@micons.cu

Recibido-Received : $2013-10-14$

Aceptado-Accepted : :2013-12-30

\section{$(\mathrm{cc}) \overline{\mathrm{EY}}$}

This work is licensed under a Creative Commons Attribution 4.0

United States License.

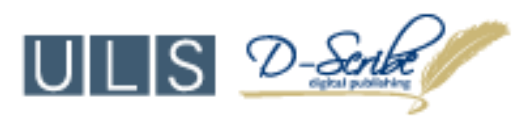

This journal is published by the University Library System of the University of Pittsburgh as part of its D-Scribe Digital Publishing Program and is cosponsored by the University of Pittsburgh Press. 